\title{
Fluid-Driven Deformation of a Soft Granular Material
}

\begin{abstract}
Christopher W. MacMinn, ${ }^{1,2, *}$ Eric R. Dufresne, ${ }^{2}$ and John S. Wettlaufer ${ }^{1,2,3}$
${ }^{1}$ University of Oxford, Oxford OX1 2JD, United Kingdom

${ }^{2}$ Yale University, New Haven, Connecticut 06520, USA

${ }^{3}$ Nordita, Royal Institute of Technology and Stockholm University, SE-10691 Stockholm, Sweden (Received 6 August 2014; revised manuscript received 4 November 2014; published 24 February 2015)

Compressing a porous, fluid-filled material drives the interstitial fluid out of the pore space, as when squeezing water out of a kitchen sponge. Inversely, injecting fluid into a porous material can deform the solid structure, as when fracturing a shale for natural gas recovery. These poromechanical interactions play an important role in geological and biological systems across a wide range of scales, from the propagation of magma through Earth's mantle to the transport of fluid through living cells and tissues. The theory of poroelasticity has been largely successful in modeling poromechanical behavior in relatively simple systems, but this continuum theory is fundamentally limited by our understanding of the pore-scale interactions between the fluid and the solid, and these problems are notoriously difficult to study in a laboratory setting. Here, we present a high-resolution measurement of injection-driven poromechanical deformation in a system with granular microsctructure: We inject fluid into a dense, confined monolayer of soft particles and use particle tracking to reveal the dynamics of the multiscale deformation field. We find that a continuum model based on poroelasticity theory captures certain macroscopic features of the deformation, but the particle-scale deformation field exhibits dramatic departures from smooth, continuum behavior. We observe particle-scale rearrangement and hysteresis, as well as petal-like mesoscale structures that are connected to material failure through spiral shear banding.
\end{abstract}

DOI: 10.1103/PhysRevX.5.011020

\section{INTRODUCTION}

Poromechanics couples the mechanical deformation of a porous solid with fluid flow through its internal structure [1-5]. In biophysics, poromechanics plays an important role in the growth and deformation of cells and tissues [6-10], and it is the dominant mechanism underlying plant motion [11]. In both pure and applied geophysics, poromechanics has been studied intensely in the context of subsurface pressurization during fluid injection, such as in geothermal energy extraction or carbon dioxide sequestration [12-15] and, particularly, in the context of hydraulic fracture for enhanced oil or gas recovery [16-18].

Poromechanical deformations are poroelastic when they are controlled by the reversible storage and release of elastic energy. The classical theory of poroelasticity couples linear elasticity with Darcy's law for fluid flow through a porous medium, and the hallmark of these systems is diffusive propagation and dissipation of fluid pressure with characteristic time scale $T_{\mathrm{pe}}=\mu L^{2} /(\mathcal{K} k)$, where $\mu$ is the

*christopher.macminn@eng.ox.ac.uk

Published by the American Physical Society under the terms of the Creative Commons Attribution 3.0 License. Further distribution of this work must maintain attribution to the author(s) and the published article's title, journal citation, and DOI.
Subject Areas: Fluid Dynamics, Mechanics, Soft Matter

viscosity of the fluid, $L$ is a characteristic length scale, and $\mathcal{K}$ and $k$ are the elastic modulus and permeability of the solid skeleton. This approach is valid for small deformations, but many real systems feature large deformations, small-scale microstructure, or physical mechanisms, such as damage, growth, or swelling, that lead to a strongly nonlinear coupling between the pore structure and the fluid flow $[5,19]$.

Many poroelastic deformations of practical interest are driven by fluid injection. Injection-driven deformations involve radial dilation (outward expansion), which is particularly interesting and challenging because it leads to a nontrivial state of stress and strain [20-23]. Indeed, fluid injection into granular materials can lead to spectacular damage patterns when the injection pressure exceeds the interparticle friction or the external confining stress [24-27]. However, the deformation in these examples is almost completely irreversible because the solid skeleton is stiff and the fluid pressure is low, so the majority of the input energy is dissipated through frictional sliding and rearrangement, and almost none is stored elastically [28]. Fluid injection can drive significant storage of elastic energy only when the fluid pressure becomes comparable to the stiffness of the solid skeleton.

True poroelastic deformation requires either much larger pressures or much softer materials. As a result, it has proven difficult to study in a laboratory setting. Experiments with rocks and sands have been limited to postmortem inspection 
after high-pressure injection $[29,30]$, providing useful insight into the failure of realistic geomaterials, but at a very coarse level in time and space. This limitation has been avoided in a one-dimensional geometry with soft, open-cell polymer foams (kitchen sponges; see, e.g., Refs. [31-33]). However, these materials have proven to be experimentally challenging for a variety of reasons, with unsatisfying comparison between experiment and theory.

Here, we study the poromechanical deformation of a system with granular microstructure by injecting fluid into a confined monolayer of spherical particles. By using particles that are extremely soft, we construct a system that exhibits striking poroelastic phenomena at relatively low working pressures. High-resolution imaging and particle tracking provide experimental access to the full, multiscale deformation field. We show that the smooth, quasireversible macroscopic deformation can be captured in part by a minimal continuum model, despite the presence of complex shear banding and structural rearrangement.

\section{FLUID INJECTION INTO A MONOLAYER OF SOFT PARTICLES}

We pack a single layer of about 25000 spherical, polyacrylamide hydrogel particles between two glass disks and saturate the packing with the working fluid, a mixture of water and glycerol [Fig. 1(a)] (see Appendix A). The disks are separated by a permeable spacer that confines the particles but allows fluid to leave freely around the edge. The particles are soft (having a Young modulus of $\sim 20 \mathrm{kPa}$ ), nearly incompressible (having a Poisson ratio of $\sim 1 / 2$ ), Hertzian (exhibiting Hertz-like contact mechanics), elastic (allowing order-one elastic strains), noncohesive, and very slippery (having low friction at particle-particle and particle-wall contacts) [34,35]. The particles have mean diameter $d \approx 1.2 \mathrm{~mm}$ with about $10 \%$ polydispersity (Fig. 5). The packing has an apparent area void fraction of $\sim 0.14$ when viewed from above, and an actual volume void fraction of $\sim 0.51$. The former is denser than random close packing in $2 \mathrm{D}(\sim 0.18)$ due in part to the softness of the particles, as often occurs in fluid emulsions, and also in part to their polydispersity.

To perform an experiment, we inject more of the same working fluid into the cell at a constant volume rate $Q$. This fluid enters the cell via an injection port in the center of the lower disk, flows radially outward through the packing, and exits through the spacer at the outer edge. The resulting fluid pressure gradient within the porous layer (large pressure at the center dropping to atmospheric at the edge) deforms the packing, driving the particles radially outward and opening a cavity in the center [Figs. 1(b) and 1(c); see also video S1 [36]]. This coupling of fluid pressure and

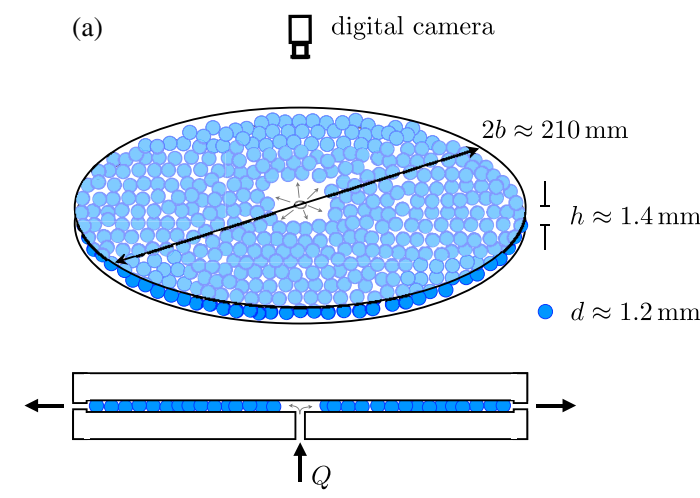

(c)

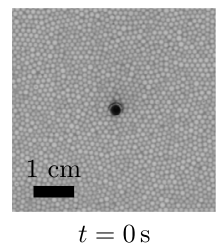

(injection starts)

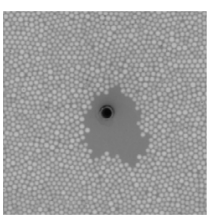

$6 \mathrm{~s}$

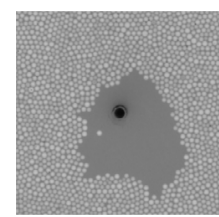

$30 \mathrm{~s}$
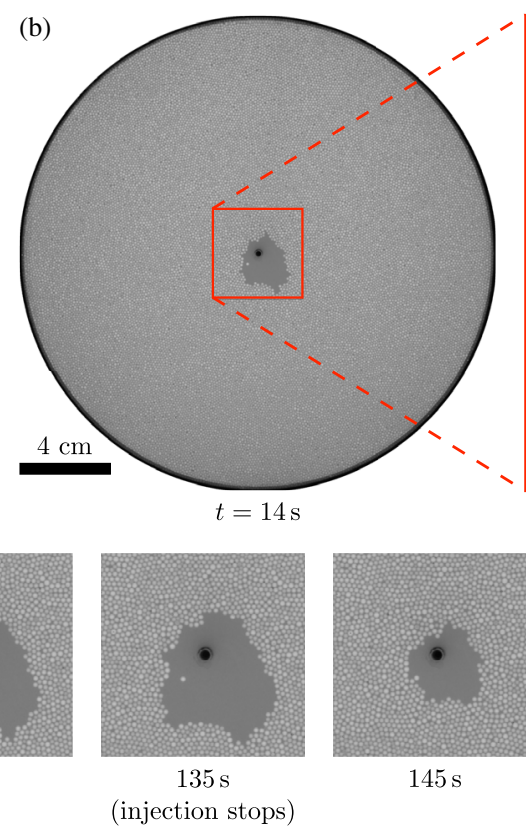

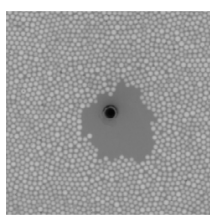

$145 \mathrm{~s}$
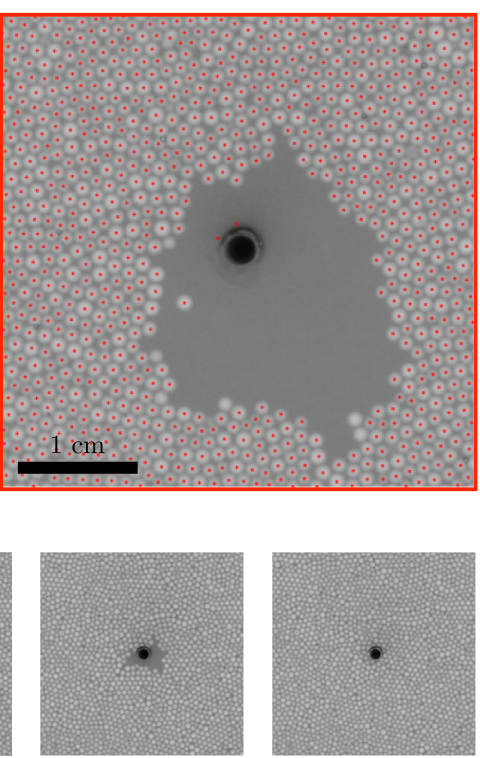

$155 \mathrm{~s}$

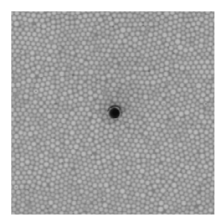

$180 \mathrm{~s}$

FIG. 1. We inject fluid into a monolayer of soft particles and measure the resulting poromechanical deformation. (a) The particles are confined between two rigid glass disks of radius $b \approx 105 \mathrm{~mm}$, and we inject fluid into the center of the packing at a steady volume rate $Q$. The fluid flows radially outward through the packing and exits along the rim through a permeable spacer. The pressure gradient due to fluid flow through the packing drives the particles radially outward, opening a cavity in the center that relaxes and closes after injection stops. (b) We image the experiment from above with a digital still camera, measuring the deformation field at high resolution by identifying and tracking the individual particles (see Appendix A). This snapshot is during injection at $Q=24 \mathrm{~mL} / \mathrm{min}$. (c) Sequence of snapshots from the same experiment showing cavity opening and relaxation. The black circle in the center is the injection port in the bottom disk, which has a diameter of about $2.5 \mathrm{~mm}$. 

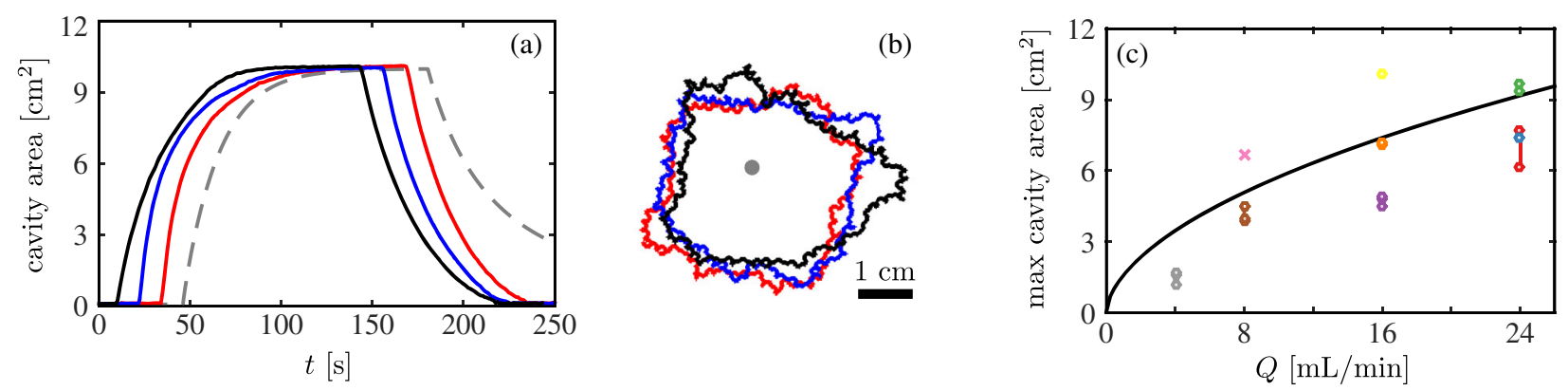

FIG. 2. Fluid injection into the center of the packing drives outward compaction, opening a cavity. When injection stops, the packing relaxes and the cavity closes. (a),(b) Here, we inject at a steady rate of $Q=16 \mathrm{~mL} / \mathrm{min}$ for about $135 \mathrm{~s}$ and then stop, allowing the packing to relax. We repeat this injection-relaxation cycle two more times (black, then blue, then red). (a) Comparing the area-versustime curves from the three cycles (offset by $12 \mathrm{~s}$ for comparison; cf. Fig. 7) shows that the process is macroscopically smooth, with similar dynamics and maximum area in each cycle. The first cycle (black) is somewhat different from subsequent cycles due to heterogeneities in the initial particle distribution. We also show the prediction of a continuum model using best-fit material properties (dashed gray curve); we discuss the model in Sec. V. (b) The steady-state cavity shape is neither smooth nor repeatable, indicating the presence of irreversible micromechanics. The cavity does not open symmetrically about the injection port (gray circle with diameter $\sim 2.5 \mathrm{~mm})$. The scalloped edges of the cavity profiles are particle-scale roughness $(\sim 1 \mathrm{~mm})$. (c) We repeat this experiment at different injection rates, showing here the maximum (i.e., steady-state) cavity area against injection rate $Q$. Each group (color) of circles indicates a series of at least two cycles after "resetting" the packing by removing the particles, cleaning the apparatus, and replacing the same particles (the cross is a single cycle). The variability between cycles is relatively small except in one case (red), indicating that cycle-tocycle irreversibilities have a weak impact on the macroscopic mechanics. The variability between series at the same injection rate is much larger, indicating that the particle arrangement has a strong impact on the macroscopic mechanics. All packings have initial porosity between 0.506 and 0.515 , and we do not observe any clear correlation between initial porosity and cavity size in this range. The black curve is the prediction of the continuum model using the same mechanical properties for all points.

solid deformation is the core idea behind poromechanics. The deformation eventually reaches a steady state (here, after $\sim 100 \mathrm{~s}$ ) in which the gradient of elastic stress in the solid skeleton balances the gradient of pressure in the fluid. We then stop injecting, at which point the elastic stress relaxes as the pressure gradient dissipates and the cavity closes. The relaxation of the packing highlights the macroscopically elastic nature of the system, demonstrating that the packing stores elastic energy during the injection phase and releases it during the relaxation phase. We repeat this injection-relaxation cycle several times in the same packing. We image the deformation and subsequent relaxation of the packing with a digital still camera, detecting the particle positions in each image to within about $0.01 \mathrm{~d}$ and then tracking the particles from image to image [Fig. 1(b)] (see Appendix A).

\section{MULTISCALE DEFORMATION FIELD}

One striking aspect of the deformation is the cavity that opens and then closes in the center of the packing. Despite the irregular shape of the cavity [Fig. 2(b)], we find that the macroscopic dynamics of its expansion and collapse are smooth and relatively reproducible across repeated injected-relaxation cycles [Figs. 2(a) and 7]. In contrast, the shape of the cavity varies from cycle to cycle [Fig. 2(b), see also video S2 [36]]. The size of the cavity increases with the injection rate roughly in accordance with the prediction of a minimal continuum model (described in more detail below), but repeating an experiment at a given injection rate after "resetting" the packing by completely rearranging the particles leads to large variability [Fig. 2(c)]. This implies that the macroscopic properties of the packing are a strong function of particle arrangement.

We measure the internal deformation of the packing via particle tracking, which provides a direct measure of the displacement field. For this purpose, we define a rectangular coordinate system centered at the injection port, where $\left(x_{i}, y_{i}\right)$ is the position of particle $i$ at time $t$ and $\left(X_{i}, Y_{i}\right)$ is its initial position. The displacement of particle $i$ is then $\boldsymbol{u}_{i}=\left(x_{i}-X_{i}, y_{i}-Y_{i}\right)$, with magnitude $u_{i}(t)=$ $\sqrt{\left(x_{i}-X_{i}\right)^{2}+\left(y_{i}-Y_{i}\right)^{2}}$ and radial component $u_{r, i}(t)=$ $\sqrt{x_{i}^{2}+y_{i}^{2}}-\sqrt{X_{i}^{2}+Y_{i}^{2}}$. The deformation is primarily radial because of the axisymmetric boundary conditions, so we focus on $u_{r}$. The difference $u-\left|u_{r}\right|$ is a measure of the nonradial component of the displacement, which we find to be a few percent or less of the radial component (see Appendix A).

We find that the radial displacement is large near the cavity and fades to zero at the rigid edge, with a petal-like mesoscale structure [Fig. 3(a); see also video S3 [36]]. Similar petal-like features have been observed in simulations of fluid injection into an initially dry packing of frictional particles [37], indicating that these structures are not an artifact of our low-friction system. Additionally, similar but much more regular features have been 

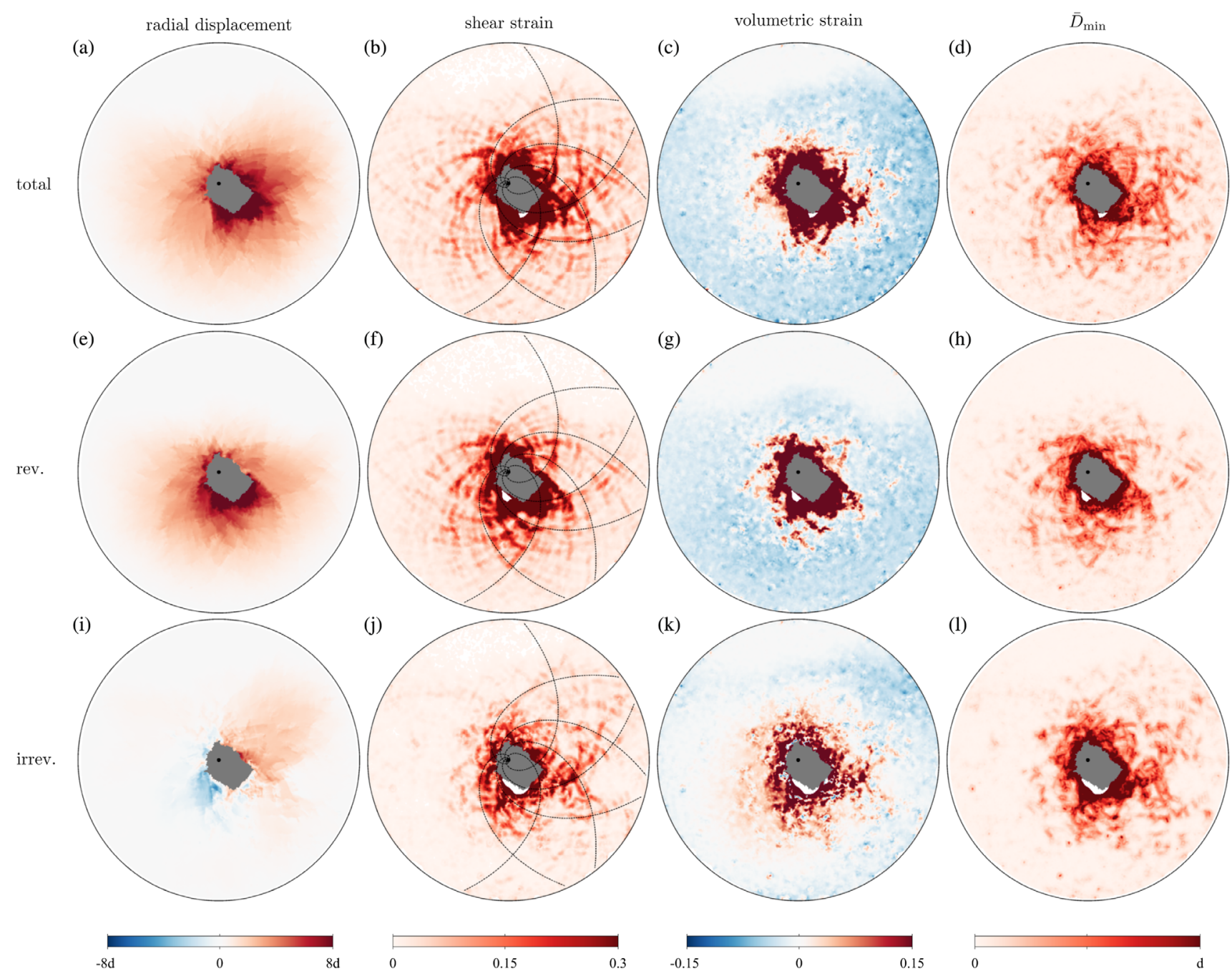

FIG. 3. The displacement field is characterized by a petal-like mesoscale structure that corresponds to spiral bands in the shear strain field. Here we show (a) the radial displacement, which reveals detailed mesoscale structure reminiscent of flower petals. We show (b) the magnitude of the shear strain with several logarithmic spirals for comparison (dashed black curves). The spirals have a pitch of $\pi / 4$, which is consistent with the shear failure of a material with negligible shear strength. We also show (c) the volumetric strain, which shows expansion in the inner region and compression in the outer region, and is relatively axisymmetric. Lastly, we include (d) the typical nonaffine displacement $\overline{\boldsymbol{D}}_{\text {min }}$ (see Appendix A). The nonaffine displacement is much smaller than the radial displacement except near the cavity, where the kinematics become strongly nonaffine. The strain field is not necessarily a good measure of the deformation in areas with large amounts of nonaffinity (near the cavity). We plot these quantities from an Eulerian perspective (against current radial position $r$ ). We decompose the deformation field into (e)-(h) reversible and (i)-(l) irreversible components. For comparison, we project all fields onto the deformed configuration at steady state. The reversible component is larger in magnitude than the irreversible component and contains most of the mesoscale structure. The irreversible component contains much of the nonaffine displacement.

observed in quasistatic "grain injection" experiments [38], where they were identified with preferential directions in the far-field crystal structure. However, our packings are isotropic due to the polydispersity of the particles.

Each petal represents a group of particles that move radially outward farther than their neighbors, implying that the edges of each petal are bands of localized shear failure. We confirm this by calculating the local strain field [39] (see Appendix A), revealing a network of spiral shear bands that span the entire system [Fig. 3(b)]. Shear bands following logarithmic spirals are a well-known feature of failure in radial dilation and hydraulic fracture [21,29]. We see spirals with a pitch of roughly of $\pi / 4$, which implies that the packing has a very low shear strength (i.e., a very low friction angle). Correlations between shear strain and positive volumetric strain are evidence of shear dilation, a well-known feature of deformation in granular materials [Figs. 3(b) and 3(c)]. 


\section{ELASTICITY, PLASTICITY, (IR)REVERSIBILITY, AND DISSIPATION}

Macroscopically, elastic deformations involve the reversible storage and release of strain energy, whereas plastic deformations are dissipative and irreversible. In crystalline solids, there is a clear distinction between elasticity and plasticity at the particle scale: Elastic deformations involve stretching or compressing bonds between atoms or molecules, whereas plastic deformations involve breaking and/ or rearranging bonds. This distinction is less clear in amorphous or granular materials, where deformations often involve a combination of reversible and irreversible rearrangement events [40-42].

Here, the fact that the cavity closes completely upon relaxation implies that the deformation is macroscopically reversible. However, the hysteresis in cavity shape is evidence of particle-scale irreversibility, and the shear bands are evidence of plastic failure. To investigate the apparent contradiction of strongly irreversible micromechanics coexisting with smooth, quasireversible macroscopic mechanics, we decompose the deformation field into reversible and irreversible components. We calculate these by considering the transformation between three configurations: the initial (before the deformation), the deformed (at steady state), and the final (relaxed, after the deformation). The total strain $\boldsymbol{E}$ is that which transforms the initial configuration into the deformed configuration. The irreversible strain $\boldsymbol{E}_{\text {irr }}$ is that which remains after the deformation relaxes (i.e., the residual strain); this transforms the initial configuration to the final relaxed configuration. The reversible strain $\boldsymbol{E}_{\text {rev }}$ is that which dissipates as the deformation relaxes; this would transform the final relaxed configuration back to the deformed configuration. For infinitesimal deformations, these three strains are related by superposition, $\boldsymbol{E}=\boldsymbol{E}_{\text {rev }}+\boldsymbol{E}_{\text {irr }}$; we calculate them independently since the deformation is large. Since we calculate strain as the locally affine best fit to the actual deformation field, we also calculate the root-mean-square difference between the affine field and the actual deformation field, $\overline{\boldsymbol{D}}_{\text {min }}$ [39] (see Appendix A). This is a measure of the typical nonaffine displacement, which is indicative of the amount of particle-level rearrangement.

Comparing the reversible and irreversible components of the strain field [Figs. 3(f) and 3(g) versus 3(j) and 3(k)], we find that the inner region is dominated by a combination of reversible and irreversible volumetric expansion (positive volumetric strain) and shear. Volumetric expansion indicates that particles have traveled away from their neighbors, which is expected near the cavity since the particles move radially outward by several diameters. Since the packing cannot support tension, this leads to local collapse or "unjamming" of the packing structure, which leads to large amounts of both reversible and irreversible rearrangement [Figs. 3(d), 3(h), and 3(l)]. In contrast, the outer region is dominated by relatively smooth, axisymmetric, reversible volumetric compression (negative volumetric strain). The displacement and shear strain are much smaller, and there is much less rearrangement. The spiral shear bands span the entire system and, surprisingly, are primarily reversible [Figs. 3(b), 3(f), and 3(g)].

All rearrangements play a strong role in the dynamics since reconfiguration of the packing takes time and dissipates energy. Macroscopically, dissipative deformations that are reversible are known as "viscoelastic," whereas those that are irreversible are known as "viscoplastic." Unlike elasticity, which is quasistatic, these viscous processes are rate dependent.

\section{POROVISCOELASTIC CONTINUUM MODEL}

The steady-state deformation is set by the balance between the gradient in fluid pressure within the packing and the roughly axisymmetric elastic compression of the outer region of the packing. Motivated by this, we next derive a minimal axisymmetric model for this system based on the theory of poroelasticity [5]. Our model is intended to capture four main features of this system: (1) conservation of volume, (2) poromechanical coupling between pressure gradients in the fluid and stress gradients in the solid skeleton, (3) elastic energy storage in the solid skeleton, and (4) viscous dissipation due to reversible and irreversible rearrangements. We do not attempt to capture the evolution of effective material properties due to irreversible rearrangements [cf. Fig. 2(c)]. We emphasize that we are not attempting to develop a general model for deforming granular materials, but rather a minimal one that captures the leading-order behavior of our poroelastic system. We outline the core assumptions of the model here and present a detailed derivation in Appendix B.

We assume that the packing is homogeneous, and that the flow and deformation fields are axisymmetric. We also assume that the fluid and the solid are individually incompressible which, for the solid, implies that the beads can rearrange and deform without changing volume. This is justified here because the working pressure is low relative to the bulk moduli of the fluid and the particles ( $\sim 5 \mathrm{kPa}$ versus $\sim 2 \mathrm{GPa}$ ). This allows for a simple but exact kinematic relationship between the volumetric strain and the local porosity (fluid or void fraction) $\phi_{f}(r, t)$ [Eq. (B10)]. We assume that the porosity is initially uniform and equal to $\phi_{f}(r, 0)=\phi_{f, 0}$.

We assume that the elastic stress in the solid skeleton is isotropic, meaning that the skeleton stores elastic energy due to volumetric compression but not due to shear. This is justified by the fact that similar packings are known to have an anomalously low ratio of shear-to-bulk modulus due to the extreme softness and slipperiness of the contacts [43]. This allows us to link the stress directly to the volumetric strain and, therefore, to $\phi_{f}$. This is useful because although the displacements are large, the changes in porosity are 
small. We take the elastic component of the stress to be a power law in the change in porosity with exponent $3 / 2$, as appropriate for a granular material consisting of Hertzian particles [44], with an effective (drained) bulk modulus $\mathcal{K}$.

The shear strain field indicates that the skeleton experiences shear failure near the cavity and along the spiral shear bands. The shear failure of granular materials is typically modeled with a frictional (Mohr-Coulomb) failure criterion, which states that the material will yield (fail plastically) when the shear stress anywhere exceeds some fixed fraction of the local normal stress. After yielding, the structure of the material rearranges according to a suitable (visco)plastic flow law. This transition to plastic flow (unjamming) has been studied extensively from a variety of perspectives (see, e.g., Refs. [39,44-46]). Although the dynamics of unjamming can be extremely important in many systems, we do not treat this behavior here because our system is compaction controlled. That is, the rate of shear strain near the cavity is fundamentally limited by the rate of volumetric strain in the outer region since the cavity can expand only as fast the outer region compresses. This is consistent with the fact that we expect the effective viscosity of rearrangement in the compacting outer region to be much larger than that in the unjamming inner region [46]. In an expansion-controlled system where the outer region is not yet jammed, we expect the cavity shape to exhibit regular, sharp, triangular cracks [47]. In contrast, we see irregular and relatively smooth cavity shapes.

We model viscous dissipation due to rearrangement in the outer region in a very simplistic way by assuming that this contributes a transient component to the volumetric stress that is linear in the rate of change of porosity with an effective viscosity $\eta$. This linear, Kelvin-Voigt-like representation is often used in viscoelasticity, but here it is intended to capture both reversible and irreversible rearrangements. The linear viscous term embodies the rate dependence of these processes, introducing a characteristic time scale for viscous rearrangement, $T_{\mathrm{vr}}=\eta / \mathcal{K}$. Although the viscosity should itself be a function of the local volumetric stress [46], we ignore this for simplicity.

Finally, we assume that fluid flows relative to the solid skeleton according to Darcy's law with a constant permeability $k$. The assumption of constant permeability is justified by the fact that the changes in porosity are relatively small, at most a few percent.

These assumptions lead to a nonlinear conservation law for the local porosity as a function of time,

$$
\frac{\partial \tilde{\phi}_{f}}{\partial \tilde{t}}+\frac{1}{\tilde{r}} \frac{\partial}{\partial \tilde{r}}\left[\alpha \tilde{\phi}_{f}-\tilde{r}\left(1-\tilde{\phi}_{f}\right) \frac{\partial \tilde{\sigma}^{\prime}}{\partial \tilde{r}}\right]=0,
$$

where $\tilde{\phi}_{f}=\left(\phi_{f}-\phi_{f, 0}\right) /\left(1-\phi_{f, 0}\right)$ is the normalized change in porosity, which is a measure of the Eulerian volumetric strain, $\tilde{t}$ and $\tilde{r}$ are time and radial position scaled by the poroelastic time scale $T_{\mathrm{pe}}=\mu b^{2} /(\mathcal{K} k)$ and the outer radius $b$, respectively, and

$$
\tilde{\sigma}^{\prime}=\tilde{\phi}_{f}\left|\tilde{\phi}_{f}\right|^{1 / 2}+\beta \frac{\partial \tilde{\phi}_{f}}{\partial \tilde{t}}
$$

is the stress in the solid skeleton (the effective stress) scaled by the bulk modulus $\mathcal{K}$. The model has two dimensionless parameters, $\alpha=\mu Q /(2 \pi h \mathcal{K} k)$ and $\beta=\eta k /\left(\mu b^{2}\right)$. The former compares the pressure gradient in the fluid with the stiffness of the solid skeleton, while the latter compares the viscous time scale to the poroelastic one. Because the mechanical properties of the packing are difficult to measure and strongly dependent on the particle arrangement, we use $\alpha$ and $\beta$ as fitting parameters. The former influences the rate of deformation and sets the steady state, whereas the latter influences only the rate.

We solve Eq. (1) subject to two boundary conditions, which are that the cavity wall is a free surface where the stress in the solid skeleton vanishes and that the outer edge is a rigid boundary where the solid is stationary. We also solve simultaneously an evolution equation for the position of the cavity wall. This model is a radial version of those that have been developed for the rectilinear deformation of kitchen sponges [33]. A similar radial model was developed in the context of blood-vessel pressurization [48], but our model is kinematically exact for finite axisymmetric deformations (see Appendix B), and we incorporate a nonlinear elastic stiffness as well as viscous dissipation.

In steady state, the model can be simplified to an integral coupled with a nonlinear algebraic equation for the cavity radius; this is straightforward to evaluate numerically. Despite the simplicity of the model and the large grainscale variability in the experiments, we find that, after choosing $\alpha$ to match the cavity area, the steady-state displacement field predicted by the model agrees surprisingly well with the azimuthal average from the experiments [Fig. 4(a)]. We also calculate an azimuthally averaged porosity field from the displacement field via Eq. (B10). The model does not agree very well with this [Fig. 4(b)], although it does capture certain aspects: The initial porosity in the experiment is $\phi_{f, 0} \approx 0.51$, and both the model and the experiment show a concave-up trend from a value near 0.51 near the cavity down to a value near 0.50 at the wall. The measured porosity field is nonmonotonic with a minimum value at $r / b \approx 0.5$, whereas the model is monotonic with its minimum at $r / b=1$, but the minimum values are similar.

The model is able to capture the displacement field relatively well because the displacement field is an integral measure of the deformation, dominated by the mean features and relatively insensitive to the local details. In contrast, the porosity field is a direct measure of the local strain, strongly reflecting the local details of the deformation. In addition, the use of Eq. (B10) assumes smoothness, 

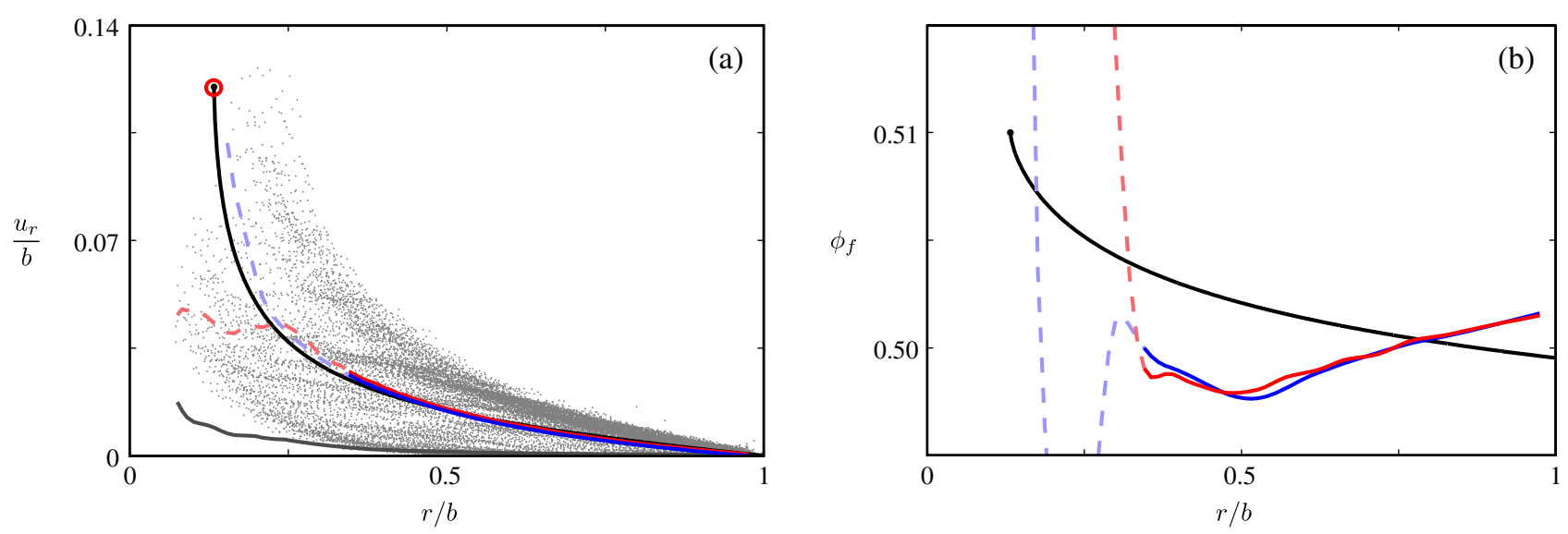

FIG. 4. Despite the large and structured variability in the particle displacements, the continuum model agrees well with the azimuthally averaged displacement profile after choosing $\alpha$ to match the cavity radius. Here we show (a) the steady-state radial displacement of the particles (gray dots), the radius of the cavity (red circle), and two different measures of the azimuthally averaged radial displacement (red curve, direct azimuthal average; blue curve, integrated volumetric strain). We also show the azimuthally averaged nonaffine displacement (solid gray curve) and the displacement field predicted by the continuum model (solid black curve). The displacement becomes strongly nonaffine near the cavity, so we choose a threshold to the left of which affine quantities are poor measures of the deformation: The dashed portions of the red and blue curves are where the nonaffine displacement accounts for more than $10 \%$ of the total. We calculate (b) two corresponding measures of the azimuthally averaged porosity by differentiating the averaged displacement according to Eq. (B10) and smoothing the result (red and blue curves). This is a poor measure of porosity near the cavity because it assumes a smooth, axisymmetric displacement field. The model (solid black curve) does not capture the porosity field very well. We plot these quantities from an Eulerian perspective.

axisymmetry, and an initially uniform porosity field, but the experiment does not necessarily observe any of these except in an averaged sense.

A more elaborate model might take advantage of plastic failure theory from soil mechanics [20], the theory of sheartransformation zones in amorphous solids [39,49], or constitutive laws from the rheology of suspensions [46], although any of these approaches would lead to a substantial increase in complexity and several additional constitutive parameters.

Solving the time-dependent model numerically, we find that it captures the dynamics of cavity expansion after choosing $\beta$ accordingly [Fig. 2(a)]. The fit $(\beta \approx 5)$ yields a poroelastic time scale of $\sim 1.5 \mathrm{~s}$ and a viscous time scale of $\sim 5 \mathrm{~s}$, implying that viscous rearrangement controls the overall rate of cavity expansion. Given the complex nature of this process, it is surprising that our linear Kelvin-Voigt model captures the dynamics of cavity expansion as well as it does. The model does not capture relaxation very well, relaxing much more slowly than the experiment. This is not surprising, since the amount of viscous dissipation due to rearrangement is likely different during relaxation than during cavity expansion. Hysteresis in effective material properties is common in plasticity, soil mechanics, and granular materials, but our minimal model does not include this.

\section{CONCLUSIONS}

Fluid injection into a soft granular material drives deformation that is macroscopically poroelastic, despite rich micromechanical complexity. We find that the deformation in the inner region (near the injection port) is dominated by irreversible structural plasticity that leads to strong variations in the cavity shape, whereas the outer region deforms smoothly and reversibly. The latter ultimately supports the radially outward loading and controls the macroscopic mechanics of the steady state, such that the leading-order features of the deformation can be captured relatively well with an axisymmetric continuum model. We expect this coexistence of microscopic irreversibility with macroscopic reversibility to be a strong feature of elastic dilational deformation in any system with a low ratio of shear strength to bulk stiffness. Many intriguing problems remain, such as connecting changes in grain-scale structure with the evolution of macroscopic properties, examining more sophisticated material models based on plasticity theory, and exploring the dynamics of fluid-driven shear failure.

More broadly, this system is a promising platform for high-resolution measurement of the dynamics of poromechanical deformation, and it has several additional features that we do not take advantage of here. For example, polyacrylamide hydrogel is closely index matched with water, making it extremely well suited to visualization in three-dimensional systems [34,35,50,51]. Polyacrylamide hydrogel is also sensitive to both temperature and dissolved salt concentration. This allows for precise system-wide tuning of the size and stiffness of the particles, and also enables studies of the dynamics of swelling or shrinking in response to local or global stimuli, which has particular relevance to biophysical systems $[7,9,11]$. 
In much the same way that granular monolayers and rafts of bubbles have served as indispensable model systems for developing fundamental concepts in the mechanics of both crystalline and amorphous materials [52-55], so too can packings of soft particles provide unique insight into the deformation and failure of materials under nontrivial poromechanical loading, from the propagation of poroelastic waves to the coupling of deformation with flow and transport. In addition to serving as a tool for benchmarking numerical simulations [37,56,57], this system offers an avenue into the experimental exploration of other fundamental problems of poroelasticity that have previously existed only as theoretical predictions or inferences from field observations.

\section{ACKNOWLEDGMENTS}

The authors gratefully acknowledge support from the Yale Climate and Energy Institute. C. W. M. thanks T. Bertrand, R. F. Katz, R. W. Style, and L. A. Wilen for helpful discussions. E. R. D. acknowledges support from the National Science Foundation (Grant No. CBET-1236086). J. S. W. acknowledges support from Yale University, the Swedish Research Council (Vetenskapsrådet), and a Royal Society Wolfson Research Merit Award.

\section{APPENDIX A: MATERIALS AND METHODS}

Apparatus.-We pack a single layer of about 25000 spherical particles (polyacrylamide hydrogel, JRM Chemical) between two borosilicate glass disks. The particles have mean diameter $d=1.19 \mathrm{~mm}$ with standard deviation (s.d.) $0.12 \mathrm{~mm}$ (10\% polydispersity; Fig. 5). The disks are $19 \mathrm{~mm}$ thick and $212.7 \mathrm{~mm}$ in diameter, and they are separated by a plastic spacer that defines a working area of diameter $b=210.5 \mathrm{~mm}$ and thickness $h=1.44 \mathrm{~mm}$. This is two s.d. greater than $d$, confining the particles to a two-dimensional monolayer without restricting their in-plane motion. The spacer is permeable, allowing fluid to exit while confining the particles. We inject fluid at a fixed volume flow rate using a syringe pump (New Era NE4000). The fluid is a mixture of water and glycerol (61\% glycerol by mass) with a viscosity of $\mu=0.012 \mathrm{Pas}$ at $20^{\circ} \mathrm{C}$. We acquire images with a digital camera (Canon EOS Rebel T2i).

Particle detection and tracking.-We process the experimental images in MATLAB, detecting particle positions in each image via centroid finding after applying an intensity threshold. We track the particles from image to image using a standard particle-tracking algorithm [58]. The images have a resolution of about 7.9 pixels per $1 \mathrm{~mm}$ (about 9.4 pixels per particle diameter). Frame-to-frame detection noise for particle centers is about 0.1 pixels $(13 \mu \mathrm{m}$ or about $1 \%$ of one diameter; Fig. 6).

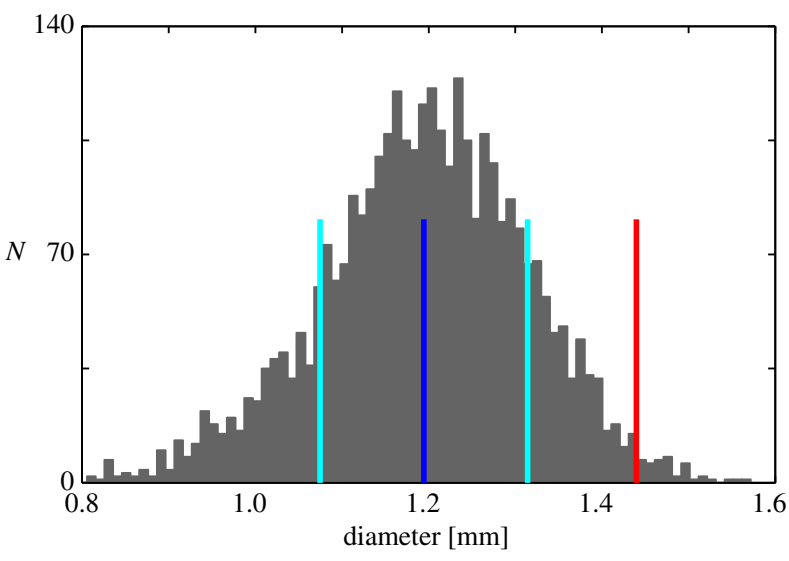

FIG. 5. The particles have mean diameter $1.19 \mathrm{~mm}$ with s.d. $0.12 \mathrm{~mm}$ (10\% polydispersity). Here, we show the particle-size distribution with the mean (vertical blue line) and the mean \pm 1 s.d. (vertical cyan lines). The gap between the glass disks is $1.44 \mathrm{~mm}$ (vertical red line), or 2 s.d. above the mean, confining the particles to a monolayer without restricting their in-plane motion. This histogram is measured optically from a sample of about 3230 particles.

Deformation field.-We use the particle positions to calculate a best-fit local strain field following Ref. [39]. However, the quantity described in Ref. [39] as a local strain tensor $\boldsymbol{\epsilon}$ is more correctly identified as a local displacement gradient tensor $\partial \boldsymbol{u} / \partial \boldsymbol{X}$, where $\boldsymbol{X}$ is the undeformed configuration and $\boldsymbol{u}$ is the displacement field. The distinction is important here, where the displacements are large. We calculate the displacement gradient tensor and then use it to calculate the Green-Lagrange strain tensor $\boldsymbol{E}=\frac{1}{2}\left(\boldsymbol{F}^{\top} \boldsymbol{F}-\boldsymbol{I}\right)$, where $\boldsymbol{F}=\boldsymbol{I}+\partial \boldsymbol{u} / \partial \boldsymbol{X}$ is the deformation gradient tensor and $\boldsymbol{I}$ is the identity tensor. The strains we report above are Green-Lagrange strains. We also calculate the root-mean-square nonaffine displacement $\overline{\boldsymbol{D}}_{\text {min }}$ from their quantity $\boldsymbol{D}_{\text {min }}^{2}$ by dividing each local value by the number of neighbors to form a mean (their quantity is a sum) and then taking the square root. The result has dimensions of length.

\section{APPENDIX B: CONTINUUM MODEL}

\section{Derivation}

We derive a continuum model for this process based on the theory of poroelasticity [5]. We assume that the fluid and the solid are incompressible, which, for the solid, implies that the beads can rearrange and deform without changing volume. This is a common assumption, justified here by the low working pressure $(\sim 5 \mathrm{kPa})$. Macroscopic deformation occurs through rearrangement of the solid skeleton, which leads to variations in the porosity (void or fluid fraction) $\phi_{f}$. Assuming axisymmetry and working strictly in terms of Eulerian quantities, conservation of mass dictates that 

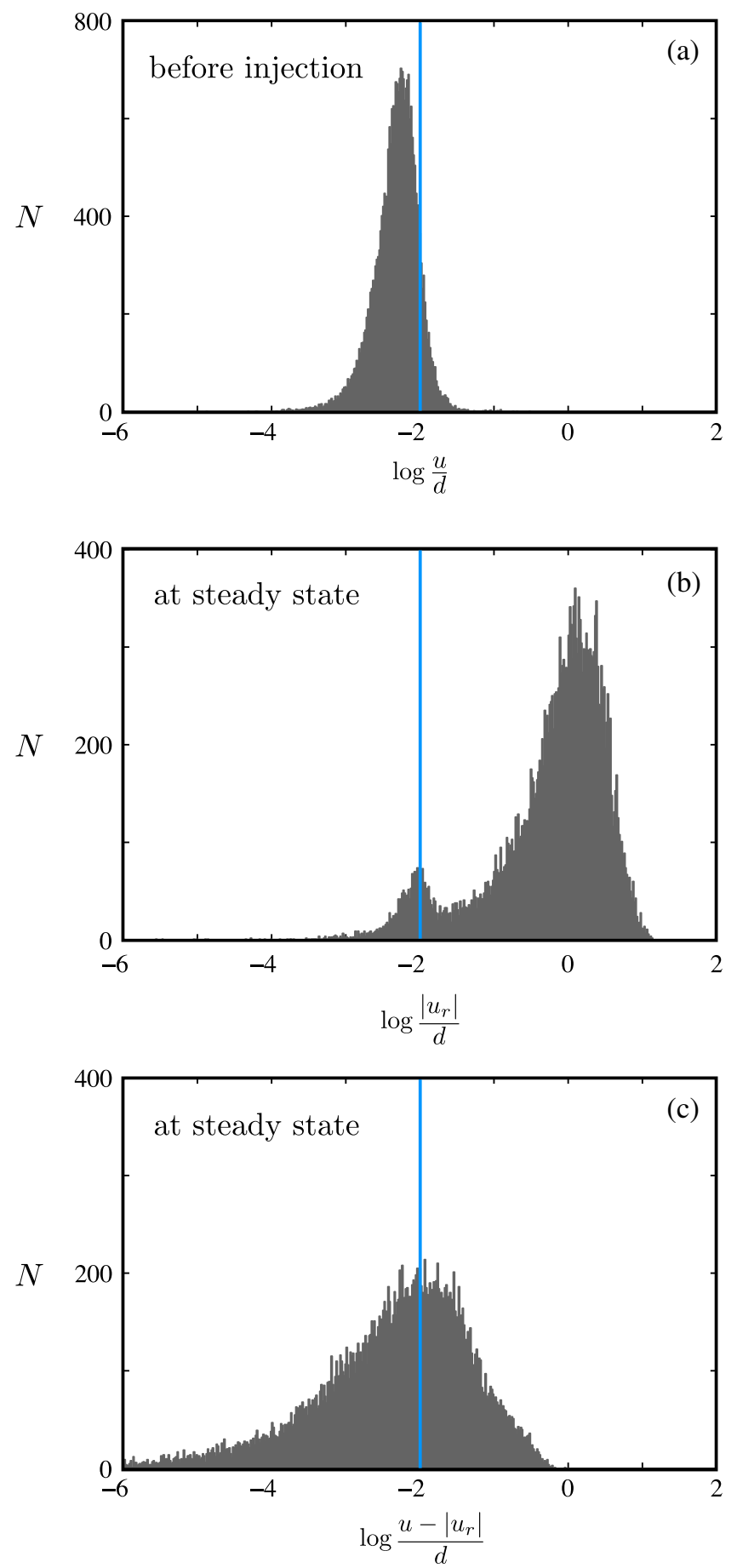

FIG. 6. We detect particle positions to within $\sim 0.01 d$. We estimate this detection noise by calculating (a) total particle displacement between two frames taken before injection has started, when the particles are at rest. The noise has mean $0.006 d$ and s.d. $0.005 d$, indicating a detection threshold of $\sim 0.01 d$ (vertical blue line). At steady-state deformation, (b) the average radial displacement is $\sim 1.4 d$ with s.d. $1.6 d$ and (c) the average nonradial displacement is $0.03 d$ with s.d. $0.06 d$, indicating that particle motion is almost entirely radial.

$$
\begin{gathered}
\frac{\partial \phi_{f}}{\partial t}+\frac{1}{r} \frac{\partial}{\partial r}\left(r \phi_{f} v_{f}\right)=0, \\
\phi_{f} v_{f}+\left(1-\phi_{f}\right) v_{s}=\frac{Q}{2 \pi r h},
\end{gathered}
$$

where $v_{f}(r, t)$ and $v_{s}(r, t)$ are the velocity of the fluid and of the solid, $Q$ is the volume rate of fluid injection, and $h$ is the thickness of the gap. We assume that fluid flows relative to the solid skeleton according to Darcy's law,

$$
\phi_{f}\left(v_{f}-v_{s}\right)=-\frac{k}{\mu} \frac{\partial p}{\partial r}
$$

where $k$ and $\mu$ are the permeability of the solid skeleton and the viscosity of the fluid, respectively, and $p(r, t)$ is the fluid pressure. Poroelastic theory dictates that the internal gradient in fluid pressure acts as a body force on the solid skeleton, and mechanical equilibrium requires that this must be supported by the divergence of the stress in the solid skeleton. We expect that the packing has an extremely low ratio of shear modulus to bulk modulus, so for simplicity we assume that the solid cannot support shear or tensile stresses. This implies that the stress tensor is isotropic, and we write mechanical equilibrium as

$$
\nabla \cdot \sigma^{\prime}=\nabla p \rightarrow \frac{\partial \sigma^{\prime}}{\partial r}=\frac{\partial p}{\partial r},
$$

where $\sigma^{\prime}$ is the effective stress (i.e., the stress in the solid skeleton). Combining all of the above, we obtain a conservation law for the evolution of the porosity,

$$
\frac{\partial \tilde{\phi}_{f}}{\partial t}+\frac{1}{r} \frac{\partial}{\partial r}\left(\frac{Q}{2 \pi h} \tilde{\phi}_{f}-r\left(1-\tilde{\phi}_{f}\right) \frac{k}{\mu} \frac{\partial \sigma^{\prime}}{\partial r}\right)=0,
$$

where $\tilde{\phi}_{f}=\left(\phi_{f}-\phi_{f, 0}\right) /\left(1-\phi_{f, 0}\right)$ is the Eulerian volumetric strain, or the normalized change in porosity, and $\phi_{f, 0}$ is the initial (relaxed) porosity.

We must now specify a constitutive relationship between effective stress and strain, but note that Eq. (B4) is valid for any stress-strain relationship that yields an isotropic stress tensor. Here, we take the stress to be Hertzian elastic and linearly viscous in the volumetric strain. This can be written as

$$
\sigma^{\prime}\left(\phi_{f}\right)=\mathcal{K} \tilde{\phi}_{f}\left|\tilde{\phi}_{f}\right|^{1 / 2}+\eta \frac{\partial \tilde{\phi}_{f}}{\partial t}
$$

where $\mathcal{K}$ and $\eta$ are the effective bulk modulus and viscosity of the solid skeleton, respectively. Note that the elastic component of the stress vanishes at $\phi_{f}=$ $\phi_{f, 0} \rightarrow \tilde{\phi}_{f}=0$. The linear viscous term introduces a 
simple "rearrangement" time scale, without which the mechanics would be quasistatic.

We assume that fluid is injected into a cavity in the solid of radius $a(t)$ and initial radius $a(0)=a_{0}$. The behavior is independent of $a_{0}$ for $a_{0} \ll b$. The fluid and the solid are initially at rest, $v_{f}(r, 0)=v_{s}(r, 0)=0$, and the initial porosity field is $\phi_{f}(r, 0)=\phi_{f, 0} \rightarrow \tilde{\phi}_{f}(r, 0)=0$. Injection begins at $t=0$.

At the inner boundary, $r=a$, the normal component of the effective stress must vanish since the cavity is a free surface of the solid skeleton. For an isotropic stress field, this implies that $\sigma^{\prime}(a, t)=0$ and, therefore, that

$$
\tilde{\phi}_{f}(a, t)=0
$$

for $\tilde{\phi}_{f}(r, 0)=0$. At the rigid outer boundary, $r=b$, we have that $u_{r}(b, t)=v_{s}(b, t)=0$. This can be written as

$$
\left.\frac{k}{\mu} \frac{\partial \sigma^{\prime}}{\partial r}\right|_{r=b}=-\frac{Q}{2 \pi b h}
$$

We also require an evolution equation for the position of the moving inner boundary,

$$
\frac{d a}{d t}=v_{s}(a, t)=\frac{Q}{2 \pi a h}+\left.\frac{k}{\mu} \frac{\partial \sigma^{\prime}}{\partial r}\right|_{r=a} .
$$

Equations (B4)-(B8) constitute a one-dimensional, timedependent, moving-boundary problem. The solid displacement field does not appear explicitly, but we can calculate it at any time through a kinematic relationship,

$$
J=\operatorname{det} \boldsymbol{F}=\frac{1-\phi_{f, 0}}{1-\phi_{f}},
$$

where $\boldsymbol{F}$ is the deformation gradient tensor (see Appendix A) and $J$ is the Jacobian determinant. For an axisymmetric, plane-strain deformation, Eq. (B9) becomes

$$
\frac{\phi_{f}-\phi_{f, 0}}{1-\phi_{f, 0}}=\tilde{\phi}_{f}=\frac{1}{r} \frac{\partial}{\partial r}\left(r u_{r}-\frac{1}{2} u_{r}^{2}\right),
$$

where $u_{r}(r, t)$ is the $r$ component of the (Eulerian) solid displacement field. In contrast to linear poroelasticity, this problem is nonlinear for four reasons: We account rigorously for (1) the moving boundary, (2) the solid velocity, and (3) the exact relationship between porosity and displacement, and we use (4) a nonlinear elastic law.

A similar axisymmetric model was developed in Ref. [48] for small elastic deformations. Our model incorporates a nonlinear elastic law and linear viscous effects and is kinematically exact — it is valid for arbitrarily large deformations as long as the constitutive laws remain valid.

\section{Dimensionless form}

We present Eqs. (B4) and (B5) in dimensionless form in the main text [Eqs. (1) and (2), respectively], where $\tilde{r}=r / b$ is the radial coordinate scaled by the outer radius, $\tilde{t}=t / T_{\mathrm{pe}}$ is time scaled by the poroelastic time scale, $\tilde{\sigma}^{\prime}=\sigma^{\prime} / \mathcal{K}$ is the effective stress scaled by the bulk modulus, and the two dimensionless parameters are $\alpha=\mu Q /(2 \pi h \mathcal{K} k)$ and $\beta=\eta k /\left(\mu b^{2}\right)$. The dimensionless boundary conditions are

$$
\begin{gathered}
\tilde{\phi}_{f}(\tilde{a}, \tilde{t})=0, \\
\left.\frac{\partial \tilde{\sigma}^{\prime}}{\partial \tilde{r}}\right|_{\tilde{r}=1}=-\alpha,
\end{gathered}
$$

and

$$
\frac{d \tilde{a}}{d \tilde{t}}=\frac{\alpha}{\tilde{a}}+\left.\frac{\partial \tilde{\sigma}^{\prime}}{\partial \tilde{r}}\right|_{\tilde{r}=\tilde{a}},
$$

where $\tilde{a}=a / b$. We solve the model numerically using a finite-volume method with explicit time integration, accommodating the moving boundary with an adaptive grid. At steady state, the model has the implicit solution

$$
\tilde{\phi}_{f, s s}=-\left[\alpha \ln \left(\tilde{r} / \tilde{a}_{s s}\right)\right]^{2 / 3}
$$

and

$$
\tilde{u}_{r, s s}(\tilde{r})=\tilde{r}-\sqrt{\tilde{r}^{2}-2 \mathcal{I}},
$$

where the integral $\mathcal{I}(\tilde{r})$ is

$$
\mathcal{I}(\tilde{r})=-\int_{\tilde{r}}^{1} r \tilde{\phi}_{f, s s}(r) d r=\int_{\tilde{r}}^{1} r\left[\alpha \ln \left(r / \tilde{a}_{s s}\right)\right]^{2 / 3} d r
$$

and the subscript " $s s$ " refers to the value of a quantity at steady state. The steady-state cavity radius is determined implicitly by the definition $\tilde{u}_{r, s s}\left(\tilde{a}_{s s}\right)=\tilde{a}_{s s}-\tilde{a}_{0}$, which leads to

$$
\tilde{a}_{s s}=\sqrt{\tilde{a}_{0}^{2}+2 \mathcal{I}\left(\tilde{a}_{s s}\right)} .
$$

We solve Eq. (B17) numerically using a standard rootfinding technique, evaluating the integral $\mathcal{I}\left(\tilde{a}_{s s}\right)$ from Eq. (B16) using the trapezoidal rule.

The mechanical properties of the packing are difficult to measure and are strongly dependent on the particle arrangement [Fig. 2(c)], so we use the mechanical properties as fitting parameters. To compare the model with the experiment at steady state, as in Fig. 4, we choose a value of the product $\mathcal{K} k$ to match the steady-state cavity radius. We use 


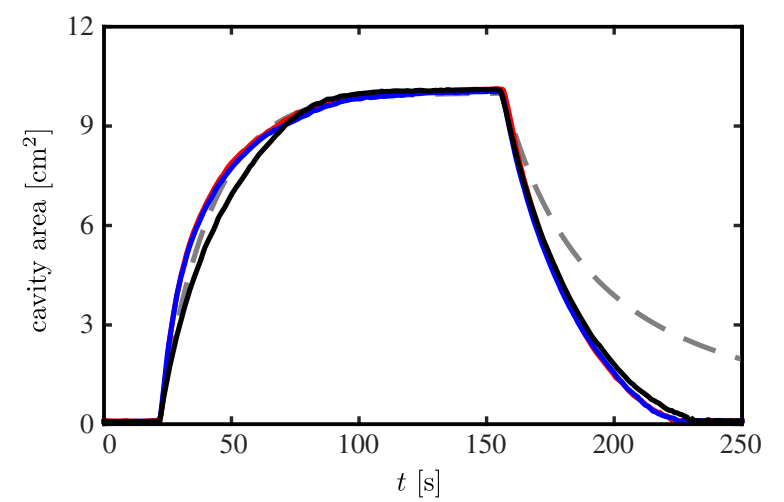

FIG. 7. Same as Fig. 2(a) but without the $12 \mathrm{~s}$ offset. The second and third cycles (blue and red curves) and the model (dashed gray curve) agree very well during cavity expansion, but the model relaxes more slowly than the experiment. The first cycle of the experiment (black) is somewhat different due to heterogeneities in the initial condition.

a single value of $\mathcal{K} k$ for all of the experiments in Fig. 2(c). To compare the dynamics, as in Figs. 2(a) and 7, we further choose $\eta k$ to match the rate of deformation and relaxation ( $\eta k$ plays no role in the steady state).

[1] K. von Terzaghi, The shearing resistance of saturated soil and the angle between the planes of shear, in Proceedings of the First International Conference on Soil Mechanics and Foundation Engineering (1936), Vol. 1, pp. 54-56.

[2] M. A. Biot, General Theory of Three-Dimensional Consolidation, J. Appl. Phys. 12, 155 (1941).

[3] R. J. Atkin and R.E. Craine, Continuum Theories of Mixtures: Applications, IMA J. Appl. Math. 17, 153 (1976).

[4] D. E. Kenyon, The Theory of an Incompressible Solid-Fluid Mixture, Arch. Ration. Mech. Anal. 62, 131 (1976).

[5] O. Coussy, Poromechanics (Wiley, New York, 2004).

[6] M. Yang and L. A. Taber, The Possible Role of Poroelasticity in the Apparent Viscoelastic Behavior of Passive Cardiac Muscle, J. Biomech. 24, 587 (1991).

[7] W. M. Lai, J. S. Hou, and V. C. Mow, A Triphasic Theory for the Swelling and Deformation Behaviors of Articular Cartilage, J. Biomech. Eng. 113, 245 (1991).

[8] S. C. Cowin, Bone Poroelasticity, J. Biomech. 32, 217 (1999).

[9] G. T. Charras, J. C. Yarrow, M. A. Horton, L. Mahadevan, and T. J. Mitchison, Non-Equilibration of Hydrostatic Pressure in Blebbing Cells, Nature (London) 435, 365 (2005).

[10] E. Moeendarbary, L. Valon, M. Fritzsche, A. R. Harris, D. A. Moulding, A. J. Thrasher, E. Stride, L. Mahadevan, and G. T. Charras, The Cytoplasm of Living Cells Behaves as a Poroelastic Material, Nat. Mater. 12, 253 (2013).

[11] J. Dumais and Y. Forterre, "Vegetable Dynamicks": The Role of Water in Plant Movements, Annu. Rev. Fluid Mech. 44, 453 (2012).

[12] M. L. Szulczewski, C. W. MacMinn, H. J. Herzog, and R. Juanes, Lifetime of Carbon Capture and Storage as a Climate-Change Mitigation Technology, Proc. Natl. Acad. Sci. U.S.A. 109, 5185 (2012).
[13] National Research Council, Induced Seismicity Potential in Energy Technologies (The National Academies Press, Washington, DC, 2013).

[14] J. P. Verdon, J.-M. Kendall, A. L. Stork, R. A. Chadwick, D. J. White, and R. C. Bissell, Comparison of Geomechanical Deformation Induced by Megatonne-Scale $\mathrm{CO}_{2}$ Storage at Sleipner, Weyburn, and In Salah, Proc. Natl. Acad. Sci. U.S.A. 110, E2762 (2013).

[15] B. Jha and R. Juanes, Coupled Multiphase Flow and Poromechanics: A Computational Model of Pore-Pressure Effects on Fault Slip and Earthquake Triggering, Water Resour. Res. 50, 3776 (2014).

[16] M. K. Hubbert and D. G. Willis, Mechanics of Hydraulic Fracturing, in Petroleum Branch Fall Meeting, Los Angeles, 1956 (AIME, Los Angeles, 1957), No. SPE-686-G, Vol. 210, pp. 153-168.

[17] E. Detournay and A. H.-D. Cheng, Poroelastic Response of a Borehole in a Non-Hydrostatic Stress Field, Int. J. Rock Mech. Min. Sci. Geomech. Abstr. 25, 171 (1988).

[18] V. M. Yarushina, D. Bercovici, and M. L. Oristaglio, Rock Deformation Models and Fluid Leak-Off in Hydraulic Fracturing, Geophys. J. Int. 194, 1514 (2013).

[19] H. F. Wang, Theory of Linear Poroelasticity (Princeton University Press, Princeton, NJ, 2000).

[20] H. S. Yu and G. T. Houlsby, Finite Cavity Expansion in Dilatant Soils: Loading Analysis, Geotechnique 41, 173 (1991).

[21] A. Alsiny, I. Vardoulakis, and A. Drescher, Deformation Localization in Cavity Inflation Experiments on Dry Sand, Geotechnique 42, 395 (1992).

[22] S. H. Hutchens and A. J. Crosby, Soft-Solid Deformation Mechanics at the Tip of an Embedded Needle, Soft Matter 10, 3679 (2014).

[23] C. Coulais, A. Seguin, and O. Dauchot, Shear Modulus and Dilatancy Softening in Granular Packings above Jamming, Phys. Rev. Lett. 113, 198001 (2014).

[24] Ø. Johnsen, R. Toussaint, K. J. Måløy, and E. G. Flekkøy, Pattern Formation during Air Injection into Granular Materials Confined in a Circular Hele-Shaw Cell, Phys. Rev. E 74, 011301 (2006).

[25] X. Cheng, L. Xu, A. Patterson, H. M. Jaeger, and S. R. Nagel, Towards the Zero-Surface-Tension Limit in Granular Fingering Instability, Nat. Phys. 4, 234 (2008).

[26] B. Sandnes, E. G. Flekkøy, H. A. Knudsen, K. J. Måløy, and H. See, Patterns and Flow in Frictional Fluid Dynamics, Nat. Commun. 2, 288 (2011).

[27] H. Huang, F. Zhang, P. Callahan, and J. Ayoub, Granular Fingering of Fluid Injection into Dense Granular Media in a Hele-Shaw Cell, Phys. Rev. Lett. 108, 258001 (2012).

[28] R. Holtzman, M. L. Szulczewski, and R. Juanes, Capillary Fracturing in Granular Media, Phys. Rev. Lett. 108, 264504 (2012).

[29] B. Bohloli and C. J. de Pater, Experimental Study on Hydraulic Fracturing of Soft Rocks: Influence of Fluid Rheology and Confining Stress, J. Pet. Sci. Eng. 53, 1 (2006).

[30] L. N. Germanovich, R. S. Hurt, J. A. Ayoub, E. Siebrits, W. D. Norman, I. Ispas, and C. Montgomery, Experimental study of hydraulic fracturing in unconsolidated materials, in SPE International Symposium and Exhibition on Formation Damage Control, Lafayette, LA, (SPE-151827-MS), 
2012, https://www.onepetro.org/conference-paper/SPE151827-MS.

[31] G. S. Beavers, T. A. Wilson, and B. A. Masha, Flow through a Deformable Porous Material, J. Appl. Mech. 42, 598 (1975).

[32] K. H. Parker, R. V. Mehta, and C. G. Caro, Steady Flow in Porous, Elastically Deformable Materials, J. Appl. Mech. 54, 794 (1987).

[33] B. Sobac, M. Colombani, and Y. Forterre, On the Dynamics of Poroelastic Foams, Mec. Ind. 12, 231 (2011).

[34] S. Mukhopadhyay and J. Peixinho, Packings of Deformable Spheres, Phys. Rev. E 84, 011302 (2011).

[35] N. Brodu, J. A. Dijksman, and R. P. Behringer, Spanning the Scales of Granular Materials: Microscopic Force Imaging, arXiv:1408.2506.

[36] See Supplemental Material at http://link.aps.org/supplemental/ 10.1103/PhysRevX.5.011020 for videos showing the experiment (S1), the particle tracking (S2), and the displacement field (S3).

[37] F. Zhang and H. Huang, Coupled DEM-CFD modeling of fluid injection into granular media, in 45th US Rock Mechanics/Geomechanics Symposium, San Francisco, CA, USA (2011) ARMA 11-228, https://www.onepetro.org/ conference-paper/ARMA-11-228.

[38] S. F. Pinto, M. S. Couto, A. P. F. Atman, S. G. Alves, A. T. Bernardes, H. F. V. de Resende, and E. C. Souza, Granular Fingers on Jammed Systems: New Fluidlike Patterns Arising in Grain-Grain invasion Experiments, Phys. Rev. Lett. 99, 068001 (2007).

[39] M. L. Falk and J.S. Langer, Dynamics of Viscoplastic Deformation in Amorphous Solids, Phys. Rev. E 57, 7192 (1998).

[40] M. Lundberg, K. Krishan, N. Xu, C. S. O'Hern, and M. Dennin, Reversible Plastic Events in Amorphous Materials, Phys. Rev. E 77, 041505 (2008).

[41] N. C. Keim and P. E. Arratia, Yielding and Microstructure in a 2D Jammed Material under Shear Deformation, Soft Matter 9, 6222 (2013).

[42] N. C. Keim and P. E. Arratia, Mechanical and Microscopic Properties of the Reversible Plastic Regime in a 2D Jammed Material, Phys. Rev. Lett. 112, 028302 (2014).

[43] J. J. Liétor-Santos, B. Sierra-Martín, and A. FernándezNieves, Bulk and Shear Moduli of Compressed Microgel Suspensions, Phys. Rev. E 84, 060402(R) (2011).
[44] C. S. O'Hern, L. E. Silbert, A. J. Liu, and S. R. Nagel, Jamming at Zero Temperature and Zero Applied Stress: The Epitome of Disorder, Phys. Rev. E 68, 011306 (2003).

[45] K. N. Nordstrom, E. Verneuil, P. E. Arratia, A. Basu, Z. Zhang, A. G. Yodh, J. P. Gollub, and D. J. Durian, Microfluidic Rheology of Soft Colloids above and below Jamming, Phys. Rev. Lett. 105, 175701 (2010).

[46] F. Boyer, É. Guazzelli, and O. Pouliquen, Unifying Suspension and Granular Rheology, Phys. Rev. Lett. 107, 188301 (2011).

[47] M. M. Bandi, T. Tallinen, and L. Mahadevan, Shock-Driven Jamming and Periodic Fracture of Particulate Rafts, Europhys. Lett. 96, 36008 (2011).

[48] S. I. Barry and G. K. Aldis, Radial Flow through Deformable Porous Shells, J. Aust. Math. Soc. Series B, Appl. Math. 34, 333 (1993).

[49] M. L. Falk and J. S. Langer, Deformation and Failure of Amorphous, Solidlike Materials, Annu. Rev. Condens. Matter Phys. 2, 353 (2011).

[50] J. A. Dijksman, F. Rietz, K. A. Lörincz, M. van Hecke, and W. Losert, Refractive Index Matched Scanning of Dense Granular Materials, Rev. Sci. Instrum. 83, 011301 (2012).

[51] M. L. Byron and E. A. Variano, Refractive-Index-Matched Hydrogel Materials for Measuring Flow-Structure Interactions, Exp. Fluids 54, 1456 (2013).

[52] L. Bragg and J. F. Nye, A Dynamical Model of a Crystal Structure, Proc. R. Soc. A 190, 474 (1947).

[53] A. S. Argon and H. Y. Kuo, Plastic Flow in a Disordered Bubble Raft (an Analog of a Metallic Glass), Mater. Sci. Eng. 39, 101 (1979).

[54] A. T. Skjeltorp and P. Meakin, Fracture in Microsphere Monolayers Studied by Experiment and Computer Simulation, Nature (London) 335, 424 (1988).

[55] P. Meakin, Models for Material Failure and Deformation, Science 252, 226 (1991).

[56] R. Holtzman and R. Juanes, Crossover from Fingering to Fracturing in Deformable Disordered Media, Phys. Rev. E 82, 046305 (2010).

[57] F. Zhang, B. Damjanac, and H. Huang, Coupled Discrete Element Modeling of Fluid Injection into Dense Granular Media, J. Geophys. Res. 118, 2703 (2013).

[58] D. L. Blair and E. R. Dufresne, The MATLAB Particle Tracking Code Repository, http://physics.georgetown.edu/matlab/. 